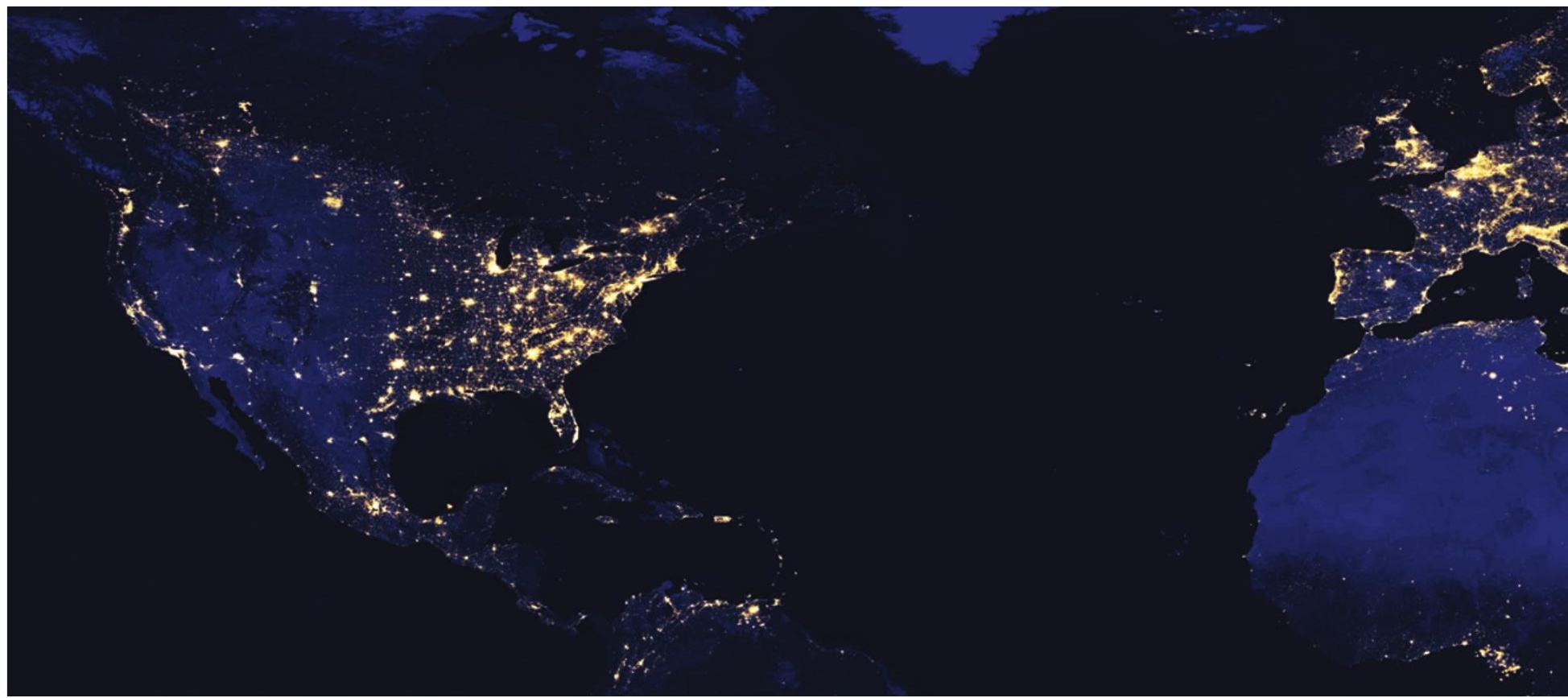

CHRONOBIOLOGY

\title{
Stepping out of time
}

\section{How can people better adapt to an 'unnatural' world of artificial lighting and alarm clocks?}

\section{BY MICHAEL EISENSTEIN}

$\mathrm{B}$ $y$ the time Elizabeth Klerman boards the train to go to her office at Brigham \& Women's Hospital in Boston, Massachusetts, the conductor has been awake for hours, rising in the dead of night for his first train at four o'clock. This schedule is not merely demanding, but contrary to most people's circadian rhythms, the pattern of physiological and metabolic activity that is roughly in synchrony with the rising and setting of the Sun.

"Some people are trying to live and work with an abnormal relationship between their circadian rhythm and the clock," says Klerman, who studies human sleep patterns. Most of us have experienced this mismatch in the form of jetlag. For shift-workers and others with 'unnatural' routines, however, this desynchronization occurs every day and can result in chronic sleep deficits. Researchers are now trying to understand the prevalence and severity of the problem, and to devise strategies that can help reset these clocks.

\section{THE TIME MACHINE}

Many body tissues have their own timetables, organized by cyclic oscillations in the expression of a network of numerous 'clock genes'. "The entire body is a clock," says Derk-Jan Dijk, director of the Surrey Sleep Research Centre in Guildford, UK. "It's a house with clocks in every room and every corner, yet in one way or another they work in an organized way." The timing of all these various 'peripheral oscillators' can profoundly affect metabolic activity, immune cell proliferation, and numerous other critical functions. But there is a central pacemaker that gives the body a sense of the time of day: the suprachiasmatic nucleus (SCN), a group of neurons in the hypothalamus (see 'The anatomy of sleep', page S2).

When melanopsin photoreceptors in the eye detect light, the SCN is activated and responds by initiating a host of rhythm-establishing physiological responses, including suppressing production of the hormone melatonin by the pineal gland (see 'The light switch'). The peripheral oscillators can be shifted by physical activity or by altering meal times, but most research suggests that light exposure is by far the most important determinant of rhythms driven by the SCN. "If you look at the data for humans, every time they suggested that exercise or food may shift the clock, they also suggest that light may have been involved," says Debra Skene, who studies chronobiology at the University of Surrey, UK.

Light is the dominant influence on circadian rhythms, but other factors can come into play. A small subset of completely blind people who lack melanopsin photoreceptors, for example, can still achieve some circadian entrainment through external cues and lifestyle ${ }^{1}$. This

timetable can be shattered by a trip across a few time zones, however, requiring long periods of readjustment without the assistance of light to signal the time of day. Many other totally blind individuals fail to entrain at all, with profound effects. "They sleep at night because that's when they're told to sleep, so they have very short sleep of poor quality, and at lunchtime their circadian system starts saying they should go to sleep," says Skene. "So we see them extremely tired - they nap and they don't perform well."

\section{BASIC INSTINCTS}

Humans are diurnal animals and so tend to be active by day and rest at night. But personal preferences for when to sleep can differ considerably among individuals, and even at different stages in the same person's life - the difference between being early birds or night owls.

Researchers are still grappling with the best approach to measure the innate timing of someone's internal clock. As an indicator of 'biological night', levels of melatonin in various body fluids can give researchers a way to monitor the SCN cycle directly in an individual. But

DNATURE.COM Scientists tinker with our circadian clocks:

go.nature.com/dqg4h this requires repeated body fluid sampling over extended periods, and is therefore impractical for population-scale studies. Instead, most sleep 


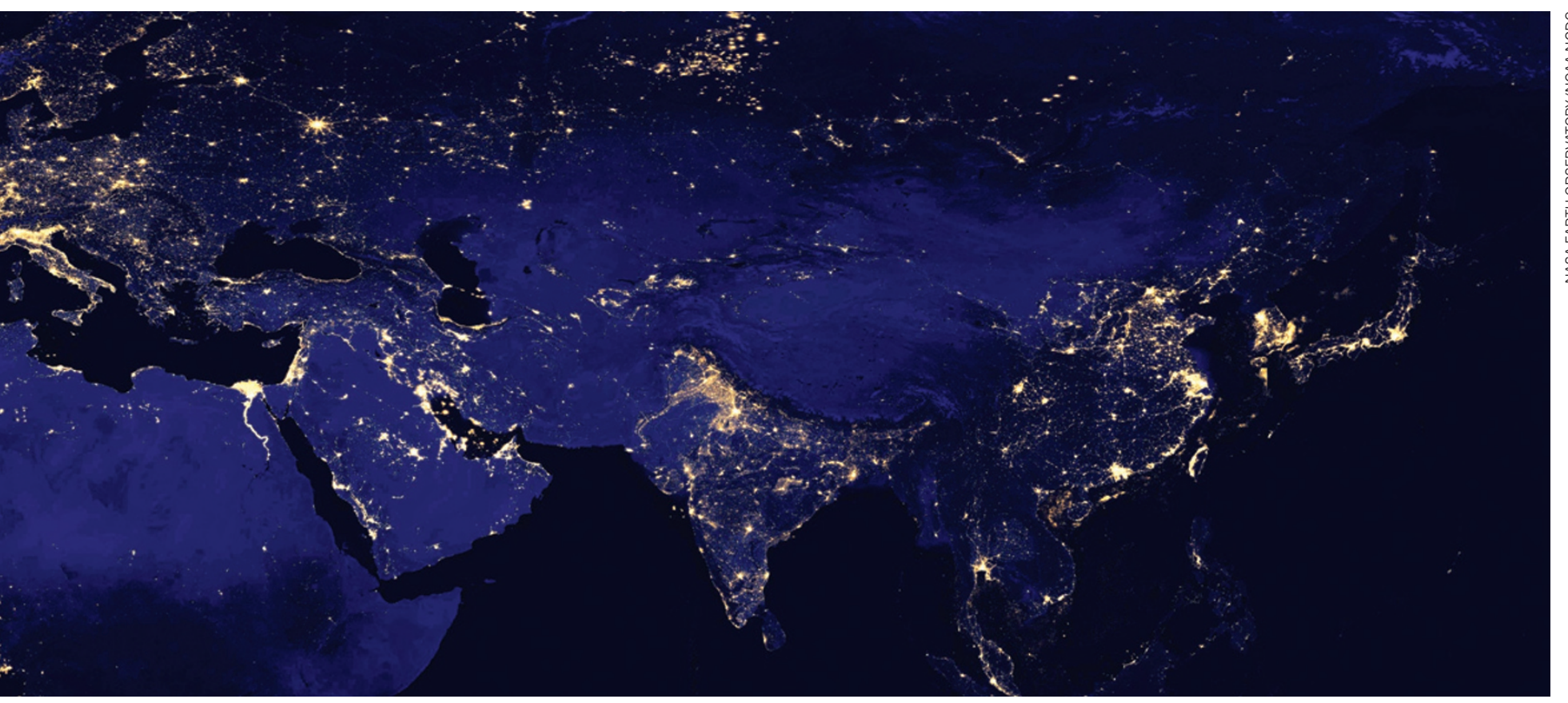

Exposure to light after sunset tricks the body into thinking it's still daytime, delaying the onset of 'biological' night. than 150,000 respondents from around the world, is the Munich Chronotype Questionnaire (MCTQ), run by Till Roenneberg at the Ludwig Maximilians University in Munich, Germany. His team devised an online survey that asks people to describe the timing of their sleep behaviour on a day-to-day basis, both on normal working or school days and at weekends or holidays. By characterizing individual sleep patterns - what Roenneberg calls a 'chronotype' - it is possible to quantify habits previously observed only at an anecdotal level, such as the tendencies of children to wake early and of teenagers to sleep late. "We were able to show how drastically the clock gets later from childhood through adolescence, reaching peak lateness in women at 19-and-a-half and in men at 21, and after those ages people get earlier again until they die," says Roenneberg.

\section{ALARM CLOCK SHOCK}

The MCTQ data have also provided insights into how our biology is altered by living and working in the artificially illuminated, industrialized world ${ }^{2}$. By assessing both rural and urban populations, Roenneberg and others have shown how modern life scatters people's sleep patterns even further around the clock. "If we were all farmers, working outside all day, chronotypes would vary only by three to four hours," says Roenneberg. "But since most of us work predominantly indoors and use artificial light after sunset, our clocks don't receive strong synchronizing signals anymore, and chronotypes nowadays span up to 12 hours."

Circadian desynchrony is most acute in people whose work schedules make them live nocturnal lives. "They are exposed to a very complex light-dark cycle, where there is artificial light at night but still some natural light that you may see during the commute home or to work," says Dijk. "In the majority of those types of shift workers, their central clock does not adapt."

Roenneberg's team has found that circadian desynchrony may be far more pervasive, however. Many modern workers effectively live on two different timetables - one enforced by their weekday alarm clock, and the other aligned to their weekend socializing and 'sleeping in' - resulting in disruption that he has dubbed 'social jetlag's. "In most people, it looks as if they were travelling from Europe to the United States on a Friday evening and back on a Monday morning, because their displacement is so large," says Roenneberg. This disconnect begins at adolescence, when our body clocks reach their latest preferred wake time, and continues all the way to retirement age.

People who operate on schedules not aligned to their internal rhythms, either due to shift work or social jetlag, often exhibit signs of chronic sleep restriction or disruption that can impair both job performance and overall wellbeing. "During wakefulness, you will have problems maintaining sustained attention," says Dijk. "You will be sleepier and experience disruption in working memory - you will see the effects across all cognitive domains."

In the long term, such desynchrony can exacerbate the risk of cardiovascular disease, obesity and other health problems ${ }^{3,4}$ (see 'Heavy sleepers', page S8). "In our animal models of 'clock gene' mutations, we're seeing diabetes and a propensity for obesity and metabolic disorders," says Joseph Takahashi, who studies circadian rhythms at the University of Texas Southwestern Medical Center in Dallas. Several studies have found a similar connection in shift workers and other individuals operating on schedules not aligned to their internal rhythms ${ }^{3,4}$. These findings "don't necessarily mean that there are immediate health consequences", says Dijk, "but we can see the impact of being asleep or being awake at the wrong phase of your circadian cycle immediately."

\section{BLUE IS THE COLOUR}

At night, artificial lighting continues to activate the SCN and disrupt the natural release of melatonin, which normally heralds the onset of biological night (see 'Casting light on sleep deficiency', page S13). But not all light stimulates the SCN equally. Skene and others have shown that specific wavelengths are especially important 'waking' signals. "We observed peak light sensitivity at a wavelength of around 460 to 480 nanometres - a nice, deep blue," she says. Red light, by contrast, has only a weak impact on melanopsin receptors and is less prone to stimulate wakefulness. So adjusting the relative levels of blue and red light that people are exposed to throughout the day could preserve normal circadian timing even during prolonged exposure to artificial light.

Klerman is collaborating with her colleague Steven Lockley at Brigham \& Women's Hospital and with George Brainard of Thomas Jefferson University in Philadelphia, Pennsylvania, to test this approach in an extreme situation: the International Space Station. Long-term isolation in cramped quarters poses many problems for astronauts, and they also experience disorienting light-dark cycles resulting from the station's orbital time of 90 minutes. "This is too short for our circadian system to synchronize," says Lockley. "The body clock starts to free run on 
its own time, just like for blind people." This is further confounded by the need to interact with people operating on various Earth schedules, such as mission control in the United States or crews arriving from Russia.

Lockley and colleagues previously showed that blue light could help to synchronize Earthbased crews with the Martian day as part of the Phoenix Mars Lander mission ${ }^{5}$. The researchers are now exploring programmable LED (lightemitting diode) systems that dynamically shift from blue-enriched to red-enriched white light on a 24-hour cycle. "We're working on shifting people's rhythms more quickly and maintaining their alertness at a better level," says Lockley.

This technology may be most valuable in extreme places such as spacecraft, submarines or Antarctic research facilities, but the broader potential is obvious. The electronics company Philips, based in Amsterdam, the Netherlands, is one of several developing controllable, dynamic lighting systems for homes, schools and offices that boost blue wavelengths early in the day and in the post-lunch slump, and shift to redder wavelengths later in the afternoon.

\section{A DOSE OF RHYTHM}

It is not yet known whether these lighting systems will be effective in groups composed of people with widely varying chronotypes, but drugs that tinker with circadian rhythms could provide a more personalized approach. The hormone melatonin may not be sufficient by itself to send someone to sleep, but it nevertheless helps the body to prepare for sleep, and there is evidence it can affect the timing of sleep. "The general consensus is that melatonin can phase-shift circadian rhythms when properly applied," says Klerman. Indeed, a growing body of work suggests that the combination of properly timed melatonin dosing and managed light exposure can counter the circadian problems associated with both jetlag and shift work ${ }^{6}$.

Melatonin requires a prescription in Europe but is available over the counter at health-food stores in the United States. However, the US Food and Drug Administration has limited oversight over the quality and content of such 'natural supplements', so it is difficult for US consumers to achieve correct dosing. As a more reliable alternative, several drug companies are developing synthetic agents that mimic the effects of melatonin, such as tasimelteon, developed by Vanda Pharmaceuticals in Washington, DC. Klerman and colleagues have shown that tasimelteon can improve sleep quality in time-shifted human subjects ${ }^{7}$, and it is now in a phase III clinical trial for use in blind individuals who lack melanopsin receptors. Such people "have recurrent jetlag", says Klerman, "so they are an ideal population that you would want to try to entrain with melatonin agonists."

Several other potential circadian modulators have been discovered in the past two years. Pharmacology researcher Thomas Burris and colleagues at the Scripps Research Institute in

\section{THE LIGHT SWITCH}

Light controls the body clock by activating

the suprachiasmatic nucleus (SCN).
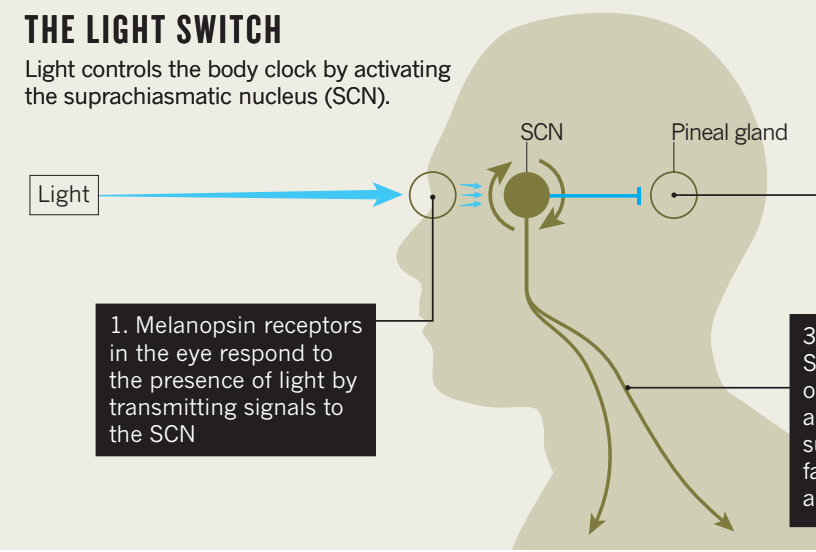

2. Light-induced activation of the SCN prevents the pineal gland from producing melatonin, a hormone that otherwise signals 'biological night'

Jupiter, Florida, identified two compounds, for example, that alter circadian rhythms by acting on a key regulator of clock gene activity. These compounds, known as SR9009 and SR9011, also affect weight gain and metabolism in mice. "Small molecules that can reset the clock might help in recovering from jetlag more rap-

\section{"I'mnot comfortable with using medication to align people to what society wants."} idly," says Takahashi, who collaborated with the Burris team and has launched a circadian drug-discovery company, Reset Therapeutics, based in Burlingame, California. $\mathrm{He}$ adds that circadian drugs could potentially treat metabolic problems associated with off-kilter body clocks, and counter the disturbed sleep that commonly afflicts elderly people.

It is less clear whether these drugs would be an appropriate solution for chronic, lifestyleassociated jetlag, however. "Medication should be used if people are sick," says Roenneberg. "I'm not comfortable with using medication to align people to what society wants." As an alternative, he recommends designing work schedules to suit individual employees and their particular chronotype, which can be determined by questionnaires such as the MCTQ.

\section{SMART SCHEDULES}

Several industries are already using smarter schedules and training methodologies that maximize the health, performance and efficiency of their workers. Major corporations such as Procter \& Gamble and Goldman Sachs are using 'sleep hygiene' programmes based on circadian research to keep their personnel sharp - for example, coaching staff to optimize their individual sleep schedules, and to switch off laptops and e-readers in advance of bedtime. The need for such efforts is especially keen in industries with round-the-clock operations particularly those where working while tired could prove fatal, such as mining or manufacturing. In aviation, the US Federal Aviation Administration has recently put in place 'fatigue risk management systems' that aim to improve the safety of air travel by using carefully regulated work schedules and mandated rest time to minimize flight-crew fatigue.

However, Lockley questions the wisdom of retraining the public to adapt to schedules that are contrary to their biological needs. "Where we have to have $24 / 7$ society - in health and safety services, for example - we should do it," he says. "But we should critically review whether we need 24-hour supermarkets or TV."

To help people make the most of their sleep while also leading happy and productive lives, we need a better sense of what natural human sleep patterns really are, and how our lifestyles reshape them. But this requires more data. Several research groups are now working with preindustrial communities in the Amazon to get a better understanding of how the natural human clock runs in a non-electric world. Meanwhile, Roenneberg hopes to build on the success of the MCTQ with a much broader Human Sleep Project that will bring together leading sleep researchers to characterize circadian rhythms and sleep patterns at the population scale.

Waking up early to start work at four oclock in the morning may never be entirely natural for people such as Klerman's train conductor, but better insights into sleep management could make such schedules more comfortable and the transition from weekend to the working week less jarring. "We need huge databases where thousands to millions of people have contributed data from their daily life," says Roenneberg. "Once we understand that, we can change our society and technology so that people can sleep in their proper, individual sleep windows."

Michael Eisenstein is a science writer based in Philadelphia, Pennsylvania.

1. Czeisler, C. A. et al. N. Engl. J. Med. 332, 6-11 (1995).

2. Roenneberg, T., Wirz-Justice, A. \& Merrow, M. J. Biol. Rhythms 18, 80-90 (2003).

3. Roenneberg, T. et al. Curr. Biol. 22, 939-943 (2012)

4. Buxton, O. M. et al. Sci. Transl. Med. 4, 129ra43 (2012).

5. Barger, L. K. et al. Sleep 35, 1423-1435 (2012)

6. Revell, V. L. \& Eastman, C. I. J. Biol. Rhythms 20, 353-365 (2005).

7. Rajaratnam, S. M. W. et al. Lancet 373, 482-491 (2009).

8. Solt, L. A. et al. Nature 485, 62-68 (2012). 